\title{
ON THE WEAK HOMOLOGICAL DIMENSION OF THE GROUP ALGEBRA OF SOLVABLE GROUPS
}

\author{
URS STAMMBACH
}

Let $K G$ denote the group algebra of the group $G$ over a field $K$ of characteristic 0 . The weak (homological) dimension of $K G$ is defined as the largest integer $q$ for which there exist $K G$-modules $B, C$ such that $\operatorname{Tor}_{q}^{K G}(B, C) \neq 0$. By $[1 ; p .352] q$ is equal to the largest integer for which there exists a $K G$-module $A$ such that $H_{q}(G, A) \neq 0$. We write w.dim $K G=q$.

The following is a corollary of the main result (Theorem 1): For a solvable group $G$ the weak dimension of $K G$ is equal to the Hirsch number $h G$ of $G$, w.dim $K G=h G$.

As an application we obtain (Theorem 5):

If $G$ is a nilpotent group of finite Hirsch number $h G$, then $h G$ is equal to the largest integer $q$ for which $H_{q}\left(G, K^{+}\right) \neq 0$, where $K^{+}$denotes the additive group of $K$ with trivial G-operation.

The starting point of our investigation was a result of $\mathrm{K}$. W. Gruenberg [3], communicated privately to the author, which relates the Hirsch number of a torsionfree nilpotent group to the cohomological dimension of the group. We would like to express our sincere thanks to Gruenberg for showing us his result.

1. The Hirsch number of $G$ and the weak dimension of $K G$

We define a class $C$ of groups as follows: The group $G$ is in $C$ if and only if there exists a series of normal subgroups $\left\{N_{i}\right\}, i=0, \ldots, k+1$

$$
1=N_{k+1} \subseteq N_{k} \subseteq \ldots \subseteq N_{1} \subseteq N_{0}=G
$$

such that the successive quotients $N_{i} / N_{i+1}$ for $i=0, \ldots, k$ are either locally finite or abelian. Note that $C$ contains all solvable groups.

Definition. Let $G$ be a group in $C$, and $\left\{N_{i}\right\}$ a series of normal subgroups with the above property. We define the Hirsch number $h G$ of $G$ to be the sum of the ranks of those quotients $N_{i} / N_{i+1}$ which are abelian. If this sum is not finite, we write $h G=\infty$.

The fact that $h G$ does not depend on the series of subgroups is well known; also, it follows immediately from our Theorem 1. (In the literature the Hirsch number is usually defined for a slightly different class of groups, see for example [4; p. 150]. However, the above definition is better for our purpose.)

TheOREM 1. For $G$ in $C$ we have $h G=$ w.dim $K G$.

We first prove a special case:

Proposition 2 [2]. Let $N$ be an abelian group of $\operatorname{rank} n$. Then w.dim $K N=n$ and $H_{n}\left(N, K^{+}\right)=K$.

Proof. We proceed by induction on $n$.

Received 23 May, 1969. Supported in part by NSF GP 7905.

[J. LoNDON MATH. Soc. (2), 2 (1970), 567-570] 
(a) Let $N$ be an abelian group of rank zero, and let $A$ be a $K N$-module. If $N$ is finite of order $|N|$, then it is well known that $|N| . H_{p}(N, A)=0$ for all $p \geqslant 1$. On the other hand $H_{p}(N, A)$ is a $K$-space. Since $K$ is a field of characteristic 0 , it follows that $H_{p}(N, A)=0$ for $p \geqslant 1$. But trivially, $H_{0}\left(N, K^{+}\right)=K$.

If $N$ is locally finite, then $N$ is the direct limit of its finitely generated, hence finite subgroups $N_{a}$. We then have for $p \geqslant 1$

$$
H_{p}(N, A)=\lim _{\rightarrow} H_{p}\left(N_{a}, A\right)=0
$$

(see $\left[1 ;\right.$ p. 125]). Of course $H_{0}\left(N, K^{+}\right)=K$.

(b) If $n \geqslant 1$, then $N$ contains at least one infinite cyclic subgroup $C$. Consider the extension $C \rightarrow N \rightarrow N / C$. Clearly, $\operatorname{rank}(N / C)=n-1$. The result is now deduced from the Hochschild-Serre spectral sequence [1; p. 350]

$$
H_{p}\left(N / C, H_{q}(C, A)\right) \Rightarrow H_{n}(N, A) \text {. }
$$

Since $C$ is a free group and since $H_{0}\left(C, K^{+}\right)=K=H_{1}\left(C, K^{+}\right)$we have w.dim $K C=1$. By induction we have w.dim $K(N / C)=n-1$. By the "maximum principle for spectral sequences" it follows that

$$
\text { w.dim } K N \leqslant \text { w.dim } K(N / C)+\text { w.dim } K C=n-1+1=n .
$$

But choosing $A=K^{+}$, we obtain $H_{n}\left(N, K^{+}\right)=H_{n-1}\left(N / C, H_{1}\left(C, K^{+}\right)\right)=K$.

Note that it follows from this proposition that $H_{n}(N, A)=A$ for any trivial $K N$-module $A$, for such a module is simply a $K$-space.

LEMMA 3. Let $N$ be a normal subgroup of $G$ with quotient group $G^{\prime}$. Suppose $N$ is abelian of rank $n$. Then to any $K G^{\prime}$-module $A$ there exists a $K G^{\prime}$-module $B$ such that $H_{n}(N, B) \doteq A$ as $K G^{\prime}$-module.

Proof: Let $B$ be any trivial $K N$-module. By Proposition 2 we have $H_{n}(N, B)=H_{n}\left(N, K^{+}\right) \otimes_{K} B=K \otimes_{K} B$. In virtue of the fact that $N$ is a normal subgroup of $G$, the group $H_{n}\left(N, K^{+}\right)=K$ has a $G^{\prime}$-action which is generally nontrivial. We shall denote the $K G^{\prime}$-module $H_{n}\left(N, K^{+}\right)$by $\tilde{K}$. The $G^{\prime}$-module structure of $\tilde{K} \otimes_{K} B$ is defined by $x(q \otimes b)=x q \otimes x b$ for $x \in G, q \in K, b \in B$. Denote by $\tilde{K}^{-1}$ the additive group of $K$ together with the "inverse" $G^{\prime}$-action, i.e., if $x \in G^{\prime}$ sends $l \in \tilde{K}$ into $q$, then $x$ sends $l \in \widetilde{K}^{-1}$ into $1 / q$. It is obvious that $\tilde{K}^{-1}$ is a $K G^{\prime}$ module. Define $B=\tilde{K}^{-1} \otimes_{K} A$. We claim that $\tilde{K} \otimes_{K}\left(\tilde{K}^{-1} \otimes_{K} A\right) \cong A$ as $K G^{\prime}$ modules. Clearly this is true as $K$-spaces; moreover

$$
x(\mathbf{l} \otimes \mathbf{l} \otimes a)=q \otimes 1 / q \otimes x a=x a .
$$

Lemma 4. Let $N$ be a normal subgroup of $G$ with quotient group $G^{\prime}$. Suppose $N$ is abelian of rank $n$ and suppose $h G^{\prime}=m$, then $\mathrm{w} \cdot \operatorname{dim} K G=m+n$.

Proof. Consider the Hochschild-Serre spectral sequence

$$
H_{p}\left(G^{\prime}, H_{q}(N, B)\right) \Rightarrow H_{p+q}(G, B) \text {. }
$$

Clearly w.dim $K G \leqslant n+m$. We show that there is a $K G$-module $B$ such that $H_{n+m}(G, B) \neq 0$. By hypothesis there is a $K G^{\prime}$-module $A$ such that $H_{m}\left(G^{\prime}, A\right) \neq 0$. Take $B$ to be the $K G^{\prime}$-module (hence also $K G$-module) defined in Lemma 3. By the "maximum principle of spectral sequences" we obtain

$$
H_{m+n}(G, B)=H_{m}\left(G^{\prime}, H_{n}(N, B)\right)=H_{m}\left(G^{\prime}, A\right) \neq 0 .
$$


Proof of Theorem 1. We proceed by induction on $k+h=l$ where $k$ is the length of the given series of subgroups of $G$, and $h=h G$ is the Hirsch number of $G$.

(a) If $l=0$, then $G$ is the trivial group.

(b) Let $l \geqslant 1$. Divide by $N$ the last nontrivial term $N_{k}$ in the given series of subgroups, and let $G^{\prime}=G / N$.

If $N$ is locally finite, it is the direct limit of its finitely generated hence finite subgroups $N_{\alpha}$. We therefore have $H_{q}(N, A)=\lim H_{q}\left(N_{\alpha}, A\right)=0$ for any $K N$-module $A$ and $q \geqslant 1$. Hence by the Hochschild-Serre spectral sequence we obtain

$$
H_{q}(G, A)=H_{q}\left(G^{\prime}, A_{N}\right) \text {. }
$$

This shows that w.dim $K G=$ w.dim $\left(G^{\prime}\right)=h\left(G^{\prime}\right)=h G$.

If $N$ is abelian of rank $n$, then we have w.dim $K G=$ w.dim $K\left(G^{\prime}\right)+n$ by Lemma 2 . Hence by induction w.dim $K G=h\left(G^{\prime}\right)+n=h G$.

Finally, if $h G=\infty$, then $G$ contains subgroups of any Hirsch number; hence w. $\operatorname{dim} K G$ cannot be finite.

Remark. The conjecture that $h G$ is also equal to the cohomological dimension of $K G$ turns out to be false: any infinite, locally finite abelian group is a counter example.

\section{Nilpotent groups.}

THEOREM 5. Let $G$ be a nilpotent group with $h G<\infty$. Then $h G$ is equal to the largest integer $q$ for which $H_{q}\left(G, K^{+}\right)$(or equivalently $H^{q}\left(G, K^{+}\right)$) is nontrivial.

Proof. In view of Theorem 1 we have to show that for $h=h G, H_{h}\left(G, K^{+}\right) \neq 0$. We prove $H_{h}\left(G, K^{+}\right)=K$ by induction on the length $k$ of the lower central series of $G$.

(a) If $G$ is abelian, Proposition 2 shows that

$$
H_{h}\left(G, K^{+}\right)=K
$$

for $h=h G$.

(b) If $G$ is not abelian denote by $G_{k}$ the last nonzero term of the lower central series of $G$. Since $\left[G, G_{k}\right]=1$, the subgroup $G_{k}$ must be contained in the centre of $G$. By the Hochschild-Serre spectral sequence

$$
H_{p}\left(G / G_{k}, H_{q}\left(G_{k}, K^{+}\right)\right) \Rightarrow H_{n}\left(G, K^{+}\right)
$$

we obtain for $p=h\left(G / G_{k}\right), q=h G_{k}$, and $h=h G$

$$
H_{h}\left(G, K^{+}\right)=H_{p}\left(G / G_{k}, H_{q}\left(G_{k}, K^{+}\right)\right)=H_{p}\left(G / G_{k}, K^{+}\right)=K,
$$

using the fact that $G_{k}$ is central, Proposition 2 and the induction assumption, respectively.

The cohomology part now follows from the remark that

$$
H^{q}\left(G, K^{+}\right)=\operatorname{Hom}_{K}\left(H_{q}\left(G, K^{+}\right), K^{+}\right) .
$$




\section{References}

1. H. Cartan, S. Eilenberg, Homological Algebra (Princeton University Press, 1956).

2. A. J. Douglas, "The weak global dimension of the group ring of abelian groups", J London Math. Soc. 36 (1961), 371-381.

3. K. W. Gruenberg, Springer Lecture Notes, to appear.

4. W. R. Scott, Group Theory (Prentice Hall, 1964).

Cornell University, Ithaca, N.Y. 14850, U.S.A.

Eidgenössische Technische Hochschule, Zürich, Switzerland. 